\title{
Verfügbarkeit phenolischer Komponenten aus Rotwein (Lemberger) und Rotem Traubensaft für den Menschen
}

\author{
Roland Bitsch ${ }^{1}$, Michael Netzel ${ }^{1,2}$, Gabi Netzel $^{1,2}$, Thomas Frank ${ }^{3}$, und Irmgard Bitsch ${ }^{4}$ \\ ${ }^{1}$ Institut für Ernährungswissenschaft der Friedrich-Schiller-Universität, 07743 Jena, Germany \\ ${ }^{2}$ CSIRO Food and Nutritional Sciences, QLD4108, Australia \\ ${ }^{3}$ Privater Gutachter, 65812 Bad Soden, Germany \\ ${ }^{4}$ Institut für Ernährungswissenschaft der Justus-Liebeig-Universität, 35392 Giessen, Germany
}

\begin{abstract}
Bioverfügbarkeit und Biokinetik von Polyphenolen aus Rotwein (Lemberger) gegenüber rotem Traubensaft wurden in einem gemischten Kollektiv mit 9 gesunden Probanden im crossover Vergleich untersucht. Nach Verabreichung einer Einzeldosis entweder von $400 \mathrm{ml}$ Rotwein oder $400 \mathrm{ml}$ rotem Traubensaft mit abgestimmtem Gesamtanthozyangehalt (27,6 mg bzw. 283,5 mg) wurde der Plasmaspiegel von Anthozyaninglukosiden ebenso wie deren Harnausscheidungsrate in regelmässigen Intervallen HPLC analytisch ermittelt. Aus den Plasmawerten wurden biokinetische Parameter wie AUC, Maximalkonzentration $\mathrm{c}_{\max }$, deren Zeitpunkt $\mathrm{t}_{\max }$ und die Halbwertszeit $\mathrm{t}_{1 / 2}$ abgeleitet. In der Harnausscheidung konnten $\mathrm{R}_{\max }$ (max. gemessene Ausscheidungsrate).deren Zeitpunkt $t_{\max , R}, A_{e}$ (Gesamtmenge ausgeschiedener Anthozyane) sowie der prozentuale Anteil der Aufnahmedosis $\mathrm{X}_{\mathrm{e}}$ erfasst werden. In der Biokinetik der plasmatischen Anthozyane waren keine Unterschiede zwischen Rotwein und rotem Traubensaft zu erkennen, dagegen übertraf die Anthozyanausscheidungsrate nach Traubensaftzufuhr mit 0,23\% der Aufnahmedosis signifikant diejenige nach Rotweinzufuhr mit 0,18\% der Aufnahmedosis. Signifikant erhöht war auch das antioxidative Potential im Plasma (TRAP-test) nach Traubensafteinnahme gegenüber Rotwein.

Es zeigte sich, dass Anthozyane aus roten Trauben zu geringem Anteil als intakte Glukoside resorbiert und als solche im Harn ausgeschieden werden. Roter Traubensaft scheint wenigstens gleich- oder sogar höherwertig gegenüber dem getesteten Rotwein zu sein im Vergleich der nachweisbaren, bioaktiven Inhaltsbestandteile.
\end{abstract}

Moderatem Weingenuss wird eine gesundheitlich protektive Wirkung beim Menschen zugesprochen. Insbesondere mit phenolischen Verbindungen im Rotwein und deren ausgeprägtem antioxidativen Potential als Schutz gegenüber oxidativer Schädigung des Körpergewebes durch Sauerstoffradikale werden diese positiven Effekte in Beziehung gesetzt [1]. In vivo Transfer und in vivo Wirkung dieser Antioxidantien aus polyphenolreichen Rotweinen ist derzeit noch kaum bekannt, jedoch bedeutsam für die Bewertung ihres gesundheitlichen Nutzens. Kontrovers diskutiert wird, inwieweit Alkohol Bioverfügbarkeit und -aktivität sowie renale Exkretion der in Rotwein dominierenden Anthozyane beeinflusst. Ziel der vorliegenden Studie war daher, Verfügbarkeit und Bioaktivität markanter Anthozyane aus heimischem Rotwein mit handelsüblichem rotem Traubensaft $\mathrm{zu}$ vergleichen.

\section{Material und methodik}

Als Versuchspersonen dienten 9 gesunde Probanden (4 $\sigma^{x}, 5$ ㅇ) im Alter von 24-34 Jahren und einem BMI bereich von 20-26. Im Rahmen einer einzentrigen, offenen Studie erhielten die Probanden nach 12 - stündigem Fasten im Crossover Verfahren eine Einzelportion von $400 \mathrm{ml}$ Rotwein (Lemberger, Geisenheimer Fuchsberg) oder die gleiche Dosis eines roten, handelsüblichen Traubensaftkonzentrats mit identischem Gesamtanthozyangehalt (279,6 mg im Rotwein; 283,5 mg im Traubensaft) (Tab. 1). Zwischen den Testphasen lag ein vierwöchiges Intervall. Jede Testperson diente als ihre eigene Kontrolle. Wein und Saft wurden mit Weissbrot und $30 \mathrm{~g}$ Käse verabreicht. Die Probanden wurden angehalten, anthozyanhaltige Nahrung oder Säfte oder Alkoholgetränke 24 Stunden vor und während der Studie zu meiden.

Entnahme von Blutproben erfolgte vor Beginn und $0,25,0,50,1,0,1,5,2$ und 3 Stunden nach Einnahme von Wein bzw. Saft. Zusätzlich wurden Urinproben unmittelbar vor Versuchsbeginn und danach in stündlichen Intervallen über einen Gesamtzeitraum von 7 Stunden gesammelt. Das Studiendesign war von der Ethikkommission der Universität Giessen bewilligt worden.

Der Anthozyangehalt in Plasma und Harn wurde nach üblicher HPLC - methodik analysiert [2-4].

Prinzip: Plasmaextraktion mit Hilfe einer ODS Solidphasenkartusche und Elution mit 0,44 M Trichloressigsäure in Methanol. Trennung der Anthozyane an

\footnotetext{
${ }^{1}$ Die Untersuchungen wurden finanziell unterstützt durch den Forschungskreis der Ernährungsindustrie (FEI) und das BM für Wirtschaft und Technologie; Projekt Nr. 12225 B.
} 
Tabelle 1. Zufuhr bioaktiver Komponenten.

\begin{tabular}{|l|c|c|}
\hline Komponente/Parameter & Roter Traubensaft (400 ml) & Rotwein (Lemberger, 400 ml) \\
\hline Anthozyangehalt gesamt (mg) & 283,5 & 279,6 \\
\hline $\begin{array}{l}\text { Antioxidative Kapazität } \\
\text { TRAP-test (mM) }\end{array}$ & 6,0 & 8,6 \\
\hline Sonstige Phenolverbindungen (mg) & 46,8 & 107,2 \\
\hline Gesamtphenole & 330,3 & 386,8 \\
\hline
\end{tabular}

Tabelle 2. Biokinetik der Anthozyane im Plasma nach Einnahme von Rotem Traubensaft bzw. Rotwein.

\begin{tabular}{|c|c|c|c|c|c|}
\hline \multirow{7}{*}{ Roter Traubensaft } & Anthozyane & $\overline{c_{\max }(\mathrm{ng} / \mathrm{ml})}$ & $\mathrm{t}_{\max }{ }^{\mathrm{a})}$ (Stunden) & $\begin{array}{l}\mathrm{AUC}(0-3 \mathrm{~h}) \\
\mathrm{ng} \cdot \mathrm{h} \cdot \mathrm{ml}^{-1}\end{array}$ & $\mathrm{t}_{1 / 2}$ \\
\hline & Zyanid.-3-glukosid & $0,42(118)$ & 0,5 & $0,6(83)$ & $1,61(23)$ \\
\hline & Delphinid.-3-glukosid & $6,12(67)$ & 0,5 & $11,9(55)$ & $1,72(27)$ \\
\hline & Malvid.-3-glukosid & $48,8(88)$ & 0,5 & $71,7(60)$ & $1,50(34)$ \\
\hline & Peonid.-3-glukosid & $27,3(51)$ & 0,5 & $49,7(36)$ & $1,63(19)$ \\
\hline & Petunid.-3-glukosid & $16,1(40)$ & 0,5 & $31,5(30)$ & $1,68(49)$ \\
\hline & Summe Anthozyane ${ }^{\mathrm{g})}$ & $100,1(64)$ & 0,5 & $168,4(42)$ & $1,83(28)$ \\
\hline \multirow{6}{*}{ Rotwein } & Zyanid.-3-glukosid & n.n. & n.n. & n.n. & n.n. \\
\hline & Delphinid.-3-glukosid & n.n. & n.n. & n.n. & n.n. \\
\hline & Malvid.-3-glukosid & $18,5(24)$ & 1,5 & $40,4(21)$ & $1,80(32)$ \\
\hline & Peonid.-3-glukosid & $12,6(16)$ & 1,5 & $30,7(18)$ & $1,83(40)$ \\
\hline & Petunid.-3-glukosid & $12,3(16)$ & 1,5 & $29,1(15)$ & $2,15(23)$ \\
\hline & Summe Anthozyane ${ }^{\mathrm{g}}$ & $42,9(16)$ & 1,5 & $100,8(14)$ & $1,99(28)$ \\
\hline
\end{tabular}

a) $=$ Median; g) = Summe der Glukoside von Zyanidin, Delphinidin, Malvidin, Peonidin, Petunidin;

n.n. = nicht nachweisbar; Datenangabe als geometrischer Mittelwert mit\% ualem Koeffizient der Varianz in Klammern.

einer Prontosil Eurobond RP-18 säule mit vorgeschalteter LiChrospher 100 RP-18 Schutzsäule und isokratische Elution mit Wasser/Azetonitril/Ameisensäure (81/10/9, $\mathrm{pH}$ 1,6). Detektion der Einzelsubstanzen bei $520 \mathrm{~nm}$ mit Photodiodenarray Detektor. Kommerziell erhältliche Standards dienten als Vergleich zur Identitätsbestimmung der getrennten Anthozyane. Das Detektionslimit der Analyten lag zwischen $0,65 \mathrm{ng} / \mathrm{ml}$ für Zyanidin-3-glukosid und $5,2 \mathrm{ng} / \mathrm{ml}$ für Malvidin-3-glukosid. Kalibrierung erfolgte durch Zusatz definierter Dosen der Standards zu neutralem Plasma bzw. Urin. Die chromatographischen Bedingungen waren identisch für Getränke, Plasma und Harn. Vor der HPLC-analyse wurden Saft und Rotwein 1/50 mit der mobilen Elutionsphase verdünnt. Traubensaft und Rotwein differierten geringfügig in ihrem Anthozyanprofil. Saft enthielt einen geringeren Gehalt an Glukosiden des Petunidins (42 vs. $91 \mu \mathrm{g} / \mathrm{ml}$ ) und Malvidins (327 vs. $461 \mu \mathrm{g} / \mathrm{ml}$ ) und einen leicht höheren Gehalt an Glukosiden des Delphinidins (124 vs. $95 \mu \mathrm{g} / \mathrm{ml}$ ) und des Peonidins (208 vs. $45 \mu \mathrm{g} / \mathrm{ml}$ ) gegenüber dem Rotwein.

Gesamtphenole wurden nach einer modifizierten Folin-Ciocalteau Methode bestimmt [5]. Prinzip: Hydrolyse konjugierter Polyphenole mit 1 M Salzsäure, lipidgebundener Polyphenole mit $2 \mathrm{M}$ Natronlauge in 75 prozentigem Methanol und anschliessende Ausfällung der Plasmaproteine mit 0,75 M Metaphosphorsäure, Zentrifugation und Extraktion des Überstands mit Azeton/Wasser (1/1). Anschliessende Analyse erfolgt mit dem Folin-Ciocalteau Reagenz. Angegeben werden die
Resultate als mg Gallussäureäquivalente. Die analysierten Anthozyanglukosidwerte in Plasma und Harn bildeten die Basis zur Berechnung biokinetischer Daten wie Maximalkonzentration $\mathrm{c}_{\max }$ (Plasma) bzw. maximale

Ausscheidungsrate $\mathrm{R}_{\max }$ (Harn) und der entsprechenden Zeitpunkte $t_{\max }$, der Fläche unter der Kurve AUC (Plasma) bzw. Ae (gesamte Anthozyanausscheidung im Harn) und der Eliminationshalbwertszeit $t_{1 / 2}$ (Plasma). Grundlage waren Kompartiment unabhängige pharmakokinetische Berechnungen nach Standardmethoden [6] mit Hilfe von WinNonlin Software (Version 3.3, Pharsight Co., Mountain View, Californien). Der Äquivalenztest mit 90\% Vertrauensbereich auf Basis eines 1 Proben t-Tests der log transformierten intraindividuellen Differenzen Dosis normalisierter Daten wurde zur Erkennung von Unterschieden und relativer Bioverfügbarkeit (Wein vs. Saft) zu Grunde gelegt. Vorhergehend wurden Distributionsvermutungen nach dem Shapiro-Wilk Test bestätigt auf Basis log normal verteilter intraindividueller Unterschiede. Signifikante Differenzen wurden bei einem P-wert 0,5 angenommen.

Ermittlung der Bioaktivität der Phenolverbindungen in Saft und Wein erfolgte anhand der antioxidativen Aktivität im Plasma mit Hilfe des TRAP-tests [7].

\section{Ergebnisse}

Pharmakokinetische Daten der einzelnen Anthozyane nach Konsum von rotem Traubensaft resp. Rotwein sind in Tab. 2 dargestellt. Bemerkenswerter Weise konnten 
Tabelle 3. Biokinetik der Anthozyane in der Harnausscheidung nach Einnahme von Rotem Traubensaft bzw. Rotwein.

\begin{tabular}{|l|l|l|l|l|l|}
\hline \multirow{5}{*}{ Roter Traubensaft } & Anthozyane & $\mathrm{R}_{\max (\mu \mathrm{g} / \text { Stunde) }}$ & $\begin{array}{l}\mathrm{t}_{\max }{ }^{\text {a) }} \\
(\mathrm{Stunden})\end{array}$ & $\mathrm{A}_{\mathrm{e}}(0-7 \mathrm{~h}) \mu \mathrm{g}$ & $\mathrm{X}_{\mathrm{e}}(0-7 \mathrm{~h}) \%$ \\
\cline { 2 - 6 } & Zyanid.-3-glukosid & $1,26(76)$ & 0,5 & $2,88(72)$ & 0,09 \\
\cline { 2 - 6 } & Delphinid.-3-glukosid & $39,6(91)$ & 0,5 & $101,9(72)$ & 0,20 \\
\cline { 2 - 6 } & Malvid.-3-glukosid & $86,7(126)$ & 0,5 & $236,3(96)$ & 0,18 \\
\cline { 2 - 6 } & Peonid.-3-glukosid & $86,0(79)$ & 0,5 & $240,5(66)$ & 0,29 \\
\cline { 2 - 6 } & Petunid.-3-glukosid & $20,2(46)$ & 0,5 & $53,4(31)$ & 0,32 \\
\cline { 2 - 6 } & Summe Anthozyaneg) & $241,4(82)$ & 0,5 & $653,6(67)$ & 0,23 \\
\hline \multirow{5}{*}{ Rotwein } & Zyanid.-3-glukosid & $0,66(117)$ & 2,5 & $1,45(67)$ & 0,06 \\
\cline { 2 - 6 } & Delphinid.-3-glukosid & $14,9(51)$ & 0,5 & $47,7(27)$ & 0,12 \\
\cline { 2 - 6 } & Malvid.-3-glukosid & $60,2(53)$ & 1,5 & $206,8(32)$ & 0,11 \\
\cline { 2 - 6 } & Peonid.-3-glukosid & $44,1(44)$ & 0,5 & $151,5(31)$ & 0,84 \\
\cline { 2 - 6 } & Petunid.-3-glukosid & $20,5(52)$ & 1,5 & $66,4(47)$ & 0,18 \\
\cline { 2 - 6 } & Summe Anthozyaneg) & $137,6(29)$ & 1,5 & $491,0(20)$ & 0,18 \\
\hline
\end{tabular}

a) $=$ Median; g) = Summe der Glukoside von Zyanidin, Delphinidin, Malvidin, Peonidin, Petunidin;

Datenangabe als geometrischer Mittelwert mit\%ualem Koeffizient der Varianz in Klammern.

die Anthozyane im Plasma ebenso auch in der Harnausscheidung nahezu ausschliesslich als Glukoside detektiert werden, wie dies auch von andern Autoren gezeigt werden konnte [8-11]. Weitere Konjugate oder freie Anthozyanidine waren nicht nachweisbar. Nach Traubensafteinnahme erreichte der geometrische Mittelwert der Maximalkonzentration $\mathrm{c}_{\max }$ der Summe der Anthozyane ca. $100 \mathrm{ng} / \mathrm{ml}$ Plasma bei einem Peakmaximum $t_{\max }$ von $1 / 2$ Stunde. Nach Rotweinzufuhr lag der entsprechende $\mathrm{c}_{\max }$-wert mit $43 \mathrm{ng} / \mathrm{ml}$ Plasma deutlich niedriger, das Peakmaximum $t_{\max }$ wurde erst nach 11/2 Stunden gemessen. Das geometrische Mittel der AUC im Plasma erreichte nach Weinzufuhr nur ca. $60 \%$ des Wertes nach Saftzufuhr. Angesichts der höheren intraindividuellen Variabilität von AUC und $\mathrm{c}_{\max }$ nach Traubensaftkonsum konnten statistisch signifikante Differenzen im Plasmaspiegel der Summe der Anthozyane zwischen beiden Getränken jedoch nicht nachgewiesen werden. Unterschiede bestanden lediglich im Zyanidinund Delphinidinglukosidgehalt, die nach Rotweinkonsum unterhalb der Nachweisgrenze lagen.

In der Harnausscheidung zeigt sich ein zum Plasmaprofil analoges Muster der resorbierten Anthozyanglukoside (Tab. 3). Die maximale Exkretionsrate $\mathrm{R}_{\max }$ konnte innerhalb von 11/2-21/2 Stunden nach oraler Zufuhr ermittelt werden. Übereinstimmende Beobachtungen finden sich auch bei andern Autoren [3,8]. Trotz grosser Variationsbreite im Ausscheidungsmuster insbesondere der Traubensaftanthozyane bestanden signifikante Differenzen in der Gesamtsumme der ausgeschiedenen Anthozyane zwischen beiden Getränken mit 0,23\% der aufgenommenen Dosis bei Traubensaft und $0,18 \%$ der Dosis bei Wein. Der höchste Anteil im Ausscheidungsmuster beider Getränke entfiel auf die Glukoside von Peonidin und Petunidin, der niedrigste auf Zyanidinglukosid. Die Harnausscheidung korrespondiert jedoch nicht unmittelbar mit der Resorptionsrate, da auch die hepar-biliäre Sekretion partiell zur Elimination beiträgt.

Der Gehalt an Gesamtpolyphenolen im Plasma stieg ebenfalls an und erreichte die Maximalkonzentration 30 Minuten nach Getränkezufuhr (Abb. 1). Im Gegensatz zu den plasmatischen Anthozyanen lag der

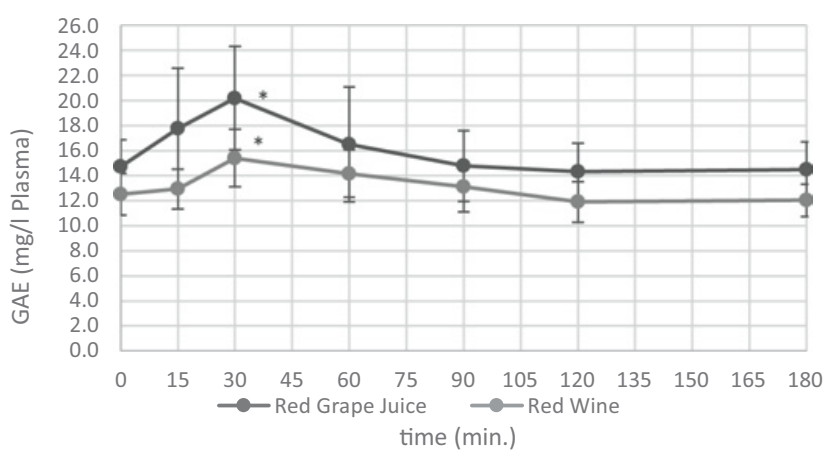

Abb. 1. Geamtphenole im Plasma (GAE = Gallussäureäquivalente) * signifikant; $\mathrm{P}<0,05$.

$\mathrm{c}_{\max }$-wert nach Traubensaftkonsum signifikant höher als die entsprechenden Werte nach Weinkonsum. Dies kann auf eine evtl. erleichterte Resorption von Flavonoiden gegenüber Anthozyanen hinweisen (s. Tab. 1), aber auch durch weitere reduzierende Inhaltsstoffe beider Getränke bedingt sein (SH-verbindungen, Askorbin-, Harnsäure etc., die zumindest partiell mit der modifizierten FolinCiocalteau Methode erfasst werden, wie auch von anderer Seite beschrieben wird [5].

Als Biomarker für die physiologische Wirksamkeit der resorbierten Polyphenole wurde zusätzlich zur Biokinetik der Anthozyane in Plasma und Harn die plasmatische antioxidative Kapazität nach Getränkekonsum mittels des TRAP - tests gemessen und die Messwerte um die jeweiligen Askorbin- und Harnsäurewerte korrigiert zur Vermeidung von Fehlinterpretationen. Wie aus Tab. 5 ersichtlich, stieg die antioxidative Kapazität nach Konsum beider Getränke an mit Maximalwerten nach 50 bzw. 63 Minuten, um nach 2 Stunden wieder auf die Ausgangswerte abzufallen. Die TRAP - biokinetik über den gemessenen Zeitraum verhielt sich analog zu den Polyphenolen. Im Falle der Traubensaftzufuhr übertrafen die gemessenen Parameter diejenigen nach Rotweinzufuhr signifikant als Hinweis einer überlegenen Bioaktivität phenolischer Komponenten insgesamt bzw. 
Tabelle 4. Biokinetik der TRAP-werte im Plasma von 9 Probanden.

\begin{tabular}{|l|l|l|l|l|l|}
\hline & $\mathrm{c}_{\max }(\mu \mathrm{Mol} / \mathrm{l})$ & $\begin{array}{l}\mathrm{c}_{\max } / \mathrm{Dosis} \\
\mu \mathrm{Mol} / 1 / \mu \mathrm{Mol}\end{array}$ & $\begin{array}{l}\mathrm{t}_{\max } \\
(\mathrm{Min} .)\end{array}$ & $\begin{array}{l}\mathrm{AUC}(0-180) \\
\mathrm{mMol} / \mathrm{Min} . / 1\end{array}$ & $\begin{array}{l}\text { AUC(0-180) } \\
\mathrm{mMol} / \mathrm{Min} . / \mathrm{mMol}\end{array}$ \\
\hline Roter Traubensaft & $1333 \pm 62$ & $0,222 \pm 0,010$ & $63 \pm 10$ & $202 \pm 11$ & $33,72 \pm 1,86$ \\
\hline Rotwein & $1233 \pm 85$ & $0,143 \pm 0,010$ & $50 \pm 15$ & $192 \pm 8$ & $22,31 \pm 0,95$ \\
\hline Signifikanz & $\mathrm{P}=0,013$ & $\mathrm{P}<0,0001$ & - & $\mathrm{P}=0,016$ & $\mathrm{P}<0,0001$ \\
\hline
\end{tabular}

Daten als arithmetrische Mittelwerte \pm Standardabweichung.

ihrer Metaboliten im roten Traubensaft gegenüber dem getesteten Rotwein.

\section{Diskussion}

Seit den Hinweisen aus dem französischen Paradoxon wird den polyphenolreichen Rotweinen ein gesundheitlich protektiver Einfluss zugeschrieben unter der Annahme, dass die intestinale Resorption von Anthzyanen durch den Alkoholgehalt des Weines erleichtert sei [1]. Gegenwärtig sind jedoch genaue Kenntnisse über die Bioverfügbarkeit dieser Verbindungen aus alkoholischen wie nicht alkoholischen Getränken unzureichend. Dies gilt in besonderem Masse auch für kinetische Daten der Resorption und Elimination dieser Polyphenole als Bezugsgrössen für vermutete gesundheitliche Wirksamkeit.

Lange Zeit herrschte die Ansicht vor, dass Anthozyane nur als freie Aglukone resorbierbar seien. Mittlerweile konnte durch unsere Gruppe wie auch von andern Autoren gezeigt werden, dass beim Menschen wie auch tierexperimentell nicht nur Monoglukoside, sondern ebenso höher kondensierte Glukoside von Fruchtsäften aus dem Intestinaltrakt ins Blut aufgenommen und im Harn als solche ausgeschieden werden [2-4,8,9,11-15]. Die im allgemeinen niedrigen Plasma- und Urinkonzentationen lassen vermuten, dass die in geringem Mass resorbierten Anthozyandosen einem raschen Metabolismus unterworfen sind, wobei auch ein Abbau bis hin zur Protokatechusäure im Plasma von Ratten nachgewiesen werden konnte nach Verfütterung einer allerdings 100 fach höheren Dosis pro $\mathrm{kg} \mathrm{KG}$ als in der vorliegenden Studie [10]. Dass die prozentual ausgeschiedene Zyanidin3-glukosiddosis in dieser Studie bei weitem tiefer lag als diejenige der andern Anthozyanglukoside kann darauf hindeuten, dass Zyanidin in vivo als Vorstufe und zu Peonidin methyliert wird, wie dies bereits tierexperimentell nachgewiesen werden konnte [10] (s. Tab. 3, X $\mathrm{X}_{\mathrm{e}}$ 0-7). Die Gesamtmenge ausgeschiedener Anthozyane kann offenbar untersuchungsbedingt grossen Schwankungen um mehr als das zehnfache unterliegen, wie auch von andern Autoren gezeigt wurde [11].

Obgleich in den Plasmakinetiken keine statistisch signifikanten Unterschiede zwischen den Getränken bestanden, lassen die entsprechenden Harnausscheidungswerte auf eine höhere Bioverfügbarkeit der Anthozyane aus rotem Traubensaft schliessen gegenüber dem getesteten Rotwein. Evtl. war der Beobachtungszeitraum relativ kurz, um eine Bioäquivalenz auch in den Plasmawerten zu erkennen

(Tab. 3, $\mathrm{R}_{\max }, \mathrm{A}_{\mathrm{e}}$ 0-7, $\mathrm{X}_{\mathrm{e}}$ 0-7). Nicht zuletzt muss aber auch berücksichtigt werden, dass infolge Adsorption an Plasmaproteine die analytische Wiederfind- ungsrate beeinträchtigt sein kann, wie von anderer Seite mit einer um 20\% geringeren Wiederfindungsrate bei mit Anthozyanextrakten aus Holunderbeeren versetzten Plasmaproben gezeigt wurde [11].

Identische Eliminationsgeschwindigkeiten $\left(t_{1}\right)$ aus dem Plasma verbunden mit einer (nicht signifikanten) Tendenz $\mathrm{zu}$ niedrigeren $\mathrm{c}_{\max }-$ und höheren $\mathrm{t}_{\max }$-werten nach Rotweinkonsum lassen darauf schliessen, dass entweder die Anthozyanresorption aus dem Rotwein verzögert oder die intestinale Aufnahme aus dem roten Traubensaft beschleunigt war (Tab. 2). Gegenwärtig sind die Befunde zum Einfluss des Alkohols auf gastrointestinale Funktionen generell und die Resorption von Flavonoiden kontrovers [15,16]. Einige Autoren konnten zeigen, dass Querzetinglukoside mit dem intestinalen Glukosetransporter SGLT-1 interagieren, wodurch die intestinale Aufnahme verbessert wird [17, 18]. Ebenso scheinen nach jüngeren Daten die intestinale Laktase-Phlorizin-Hydrolase (LPH) in der apikalen Membran intestinaler Epithelzellen und die zytosolische $\beta$-Glukosidase bei Resorption und Metabolismus von Flavonoiden eine nicht unwichtige Rolle zu spielen $[19,20]$. Unter Berücksichtigung des Zuckergehalts des getesteten Traubensafts und der Strukturähnlichkeit zu Querzetin andererseits ist es denkbar, dass die Anthozyane via Kotransport mit dem intestinalen, membrangebundenen SGLT-1 resorbiert wurden mit dem Ergebnis einer erhöhten Aufnahme aus dem Intestinaltrakt gegenüber dem verwendeten Rotwein [21].

Erhärtet wird diese Annahme durch die signifikant erhöhte Biokinetik der plasmatischen Polyphenole nach Traubensaftaufnahme (Abb. 1 oder Tab. 4). Eine höhere Bioaktivität des roten Traubensafts gegenüber Rotwein lässt sich nicht zuletzt auch der antioxidativen Kapazität im Plasma entnehmen (Tab. 5). Die $c_{\max }$-werte wie auch die AUC der gemessenen TRAP - biokinetik im Plasma waren nach Traubensaftaufnahme signifikant erhöht verglichen mit Rotweinaufnahme.

\section{Schlussfolgerung}

Die Resultate vorliegender Studie zeigen, dass die Anthozyane der roten Traube in geringem Umfang resorbiert und renal als intakte Glukoside ausgeschieden werden. Entgegen bisheriger Ansicht werden die ausgesprochen antioxidativ wirksamen Anthozyane offenbar aus Taubensaft besser resorbiert als aus Rotwein. Dementsprechend war die antioxidative Aktivität im Plasma (TRAP - test) bei menschlichen Probanden nach Traubensaftkonsum stärker angestiegen als nach Rotweinkonsum. Aus den Resultaten lässt sich die hypothetische Schlussfolgerung ziehen, dass die Anthozyanresorption 
durch den Glukosegehalt des Traubensafts entscheidend verbessert wird. Ein potentieller Alkoholeinfluss auf intestinale Aufnahme und Metabolismus bedarf weiterer Aufklärung. Nichtsdestoweniger können natürlich auch weitere Komponenten bei der Weinbereitung mit der Anthozyanresorption in Wechselwirkung treten.

\section{Literatur}

[1] S. Renaud, M. de Lorgeril, Lancet 339 (8808), 1523 (1992)

[2] T. Frank, M. Netzel, G. Strass, R. Bitsch, I. Bitsch, Can. J. Physiol. Pharmacol. 81(5), 423 (2003)

[3] T. Miyazawa, K. Nakagawa, M. Kudo, K. Muraishi, K. Someya, J. Agric. Food Chem. 47(3), 1083 (1999)

[4] M. Netzel, G. Strass, M. Janssen, I. Bitsch, R. Bitsch, J. Environ Pathol. Toxicol. Oncol. 20(2), 89 (2001)

[5] M. Serafini, G. Maiani, A. Ferro-Luzzi, J. Nutr. 128(6), 1003 (1998)

[6] W. Cawello, Parameters for Compartmet-Free Pharmacokinetics (Aachen, Shaker 1999)

[7] A. Ghiselli, M. Serafini, G. Maiani, E. Azzini, A. Ferro-Luzzi, Free Radic. Biol. Med. 18(1), 29 (1995)

[8] H. Matsumoto, H. Inaba, M. Kishi, S. Tominaga, M. Hirayama, T. Tsuda, J. Agric. Food Chem. 49(3), $1546(2001)$
[9] G. Cao, H.U. Muccitelli, C. Sanchez-Moreno, R.L. Prior, Am. J. Clin. Nutr. 73(5), 920 (2001)

[10] T. Tsuda, F. Horio, T. Osawa, FEBS Lett. 449(2-3), 179 (1999)

[11] M. Murkovic, U. Adam, W. Pfannhauser, Fresenius J. Anal. Chem. 366(4), 379 (2000)

[12] U. Mülleder, M. Murkovic, W. Pfannhauser, J. Biochem. Biophys. Methods 53(1-3), 61 (2002)

[13] X. Wu, G. Cao, R.L. Prior, J. Nutr. 132(7), 1865 (2002)

[14] M. Murkovic, U. Mülleder, U. Adam, W. Pfannhauser, J. Sci. Food Agric. 81(9), 934 (2001)

[15] A. Bub, B. Watzl, D. Heeb, G. Rechkemmer, K. Briviba, Eur. J. Nutr. 40(3), 113 (2001)

[16] D. Levanon, B. Goss, J.D.Z. Chen, Dig. Dis. Sci. 47(11), 2500 (2002)

[17] J.M. Gee, M.S. Dupont, A.J. Day, G.W. Plumb, G. Williamson, I.T. Johnson, J. Nutr. 130(11), 2765 (2000)

[18] P.C.H. Hollman, J. Sci. Food Agric. 81(9), 842 (2001)

[19] A.J. Day, F.J. Canada, J.C. Diaz et al., FEBS Lett. 468(2-3), 166 (2000)

[20] K. Németh, G.W. Plumb, J.G. Berrin et al., Eur. J. Nutr. 42(1), 29 (2003)

[21] R.P. Ferraris, Biochem. J. 360 (pt 2), 265 (2001) 